\title{
Thermal and flame retardancy behavior of oil palm based epoxy nanocomposites
}

\begin{abstract}
The aim of present study was to investigate the thermal properties and flame retardancy behavior of flame retardant (FR) epoxy nanocomposites from chemically treated (bromine water and tin chloride) oil palm empty fruit bunch (OPEFB) nano filler at different filler loading $(1,3,5 \%)$. Thermal properties were evaluated through thermogravimetry analyzer, derivative thermogravimetry and differential scanning calorimetry. FR properties of nanocomposites are evaluated through UL-94 vertical burning test and limiting oxygen index (LOI). The functional group analysis of all composites was made by FTIR spectroscopy. Thermal analysis shows that degradation temperature of epoxy composites shifts from 370 to $410{ }^{\circ} \mathrm{C}$ and char yield also increases for $3 \%$ loading. Furthermore LOI value of $29 \%$ and UL94 rating of $\mathrm{V}-0$ with no flame dripping and cotton ignition, revealed that $3 \%$ oil palm nano filler filled epoxy nanocomposites display satisfactory flame retardancy. The superior flame retardancy of epoxy nanocomposites are attributed to the chemical reactions occurred in the gaseous phases and the profound synergistic flame retardation effect of tin with bromine in the treated nano OPEFB filler. All the epoxy nanocomposites displayed almost similar FTIR spectra with the characteristics metal-halogen bond supporting the synergism. Homogeneous dispersion of $3 \%$ oil palm nano filler act as highly effective combustion chain terminating agent compared with 1 and 5\% nano OPEFB/epoxy nanocomposites.
\end{abstract}

Keyword: Epoxy; Oil palm nano filler; Nanocomposites; Thermal properties; Flame retardants 